\title{
Agronomic Journal
}

\section{UNIFORMIDADE DE APLICAÇÃO DE LÂMINA DE IRRIGAÇÃO EM CANA-DE-AÇÚCAR POR AUTOPROPELIDO COM DIFERENTES PRESSÕES DE SERVIÇO}

\author{
UNIFORMITY OF APPLICATION OF IRRIGATION BLADE IN SUGARCANE BY SELF-PROPELLED WITH \\ DIFFERENT SERVICE PRESSURES
}

Gustavo Luis Souza ${ }^{1}$, Ana Cláudia Oliveira Sérvulo ${ }^{2}$, Manoel Henrique Reis De Oliveira ${ }^{3}$, Dyb Youssef Bittar $^{2}$

${ }^{1}$ Graduado em Agronomia - Faculdade Evangélica de Goianésia

${ }^{2}$ Docente do Curso de Agronomia - Faculdade Evangélica de Goianésia

${ }^{3}$ Docente do Curso Técnico - Itego

\section{Info}

Recebido: 09/2019

Publicado: 11/2019

ISSN: 2595-6906

\section{Palavras-Chave}

Coeficiente de uniformidade

estatístico, Coeficiente de

uniformidade de Christiansen,

Coeficiente de uniformidade de

distribuição, Deriva, eficiência de

aplicação.

Keywords:

Statistical uniformity coefficient, Christiansen uniformity coefficient, Distribution uniformity coefficient, Drift, Application efficiency.

\begin{abstract}
A irrigação na cultura da cana-de-açúcar é importante no estabelecimento inicial do canavial ou salvamento após o corte. A uniformidade de aplicação da lâmina de irrigação é um critério de avaliação do sistema de irrigação e serve de base para tomada de decisão a respeito da manutenção e operação do sistema. 0 objetivo deste trabalho foi avaliar a uniformidade da lâmina aplicada por um sistema de aspersão autopropelido com diferentes pressões de serviço. 0 ensaio foi conduzido no dia 14 de setembro de 2018, no município de Santa Isabel - GO. Foram instalados copos coletores de precipitação no espaçamento entre os dois carretéis irrigadores, espaçados em $5 \mathrm{~m}$ entre coletor e $5 \mathrm{~m}$ entre linhas, totalizando 13 coletores por linha e 3 linhas de coleta. A velocidade de recolhimento foi definida em $100 \mathrm{~m} / \mathrm{h}$, na intenção de aplicar uma lâmina de 16,33 mm. 0 sistema operou em três pressões de serviço: 4,0 $\mathrm{kgf} \mathrm{cm}-2$, 5,0 kgf $\mathrm{cm}-2$ e 6,0 kgf $\mathrm{cm}-2$. Determinou-se os coeficientes de uniformidade estatístico (CUE), de Christiansen (CUC) e de distribuição (CUD), e a eficiência de aplicação (Eap).
\end{abstract} Foram monitoradas a velocidade do vento e a temperatura instantânea do ar com anemômetro digital. A uniformidade de aplicação variou entre 71,6 e 82,0\%, sendo considerada razoável. A Eap média foi de 87,1\%. A pressão de 6,0 kgf cm-2 proporcionou melhor desempenho em uniformidade e eficiência.

\section{Resumo}

Irrigation in the sugarcane crop is important in the initial establishment of the cane field or salvage after cutting. The uniformity of application of the irrigation blade is an evaluation criterion of the irrigation system and serves as a basis for decision making regarding the maintenance and operation of the system. The objective of this work was to evaluate the uniformity of the blade applied by a self-propelled sprinkler system with different service pressures. The trial was conducted on September 14, 2018, in the municipality of Santa Isabel - GO. Precipitation collector cups were installed in the spacing between the two irrigation spools, spaced $5 \mathrm{~m}$ between collector and $5 \mathrm{~m}$ between lines, totaling 13 collectors per line and 3 collection lines. The pick-up speed was set at $100 \mathrm{~m} / \mathrm{h}$, with the intention of applying a $16.33 \mathrm{~mm}$ blade. The system operated at three service pressures: $4.0 \mathrm{kgf} \mathrm{cm}-2,5.0 \mathrm{kgf} \mathrm{cm}-2$ and $6.0 \mathrm{kgf}$ cm-2. Statistical uniformity (CUE), Christiansen (CUC) and distribution (CUD) coefficients and application efficiency (Eap) were determined. Wind speed and instant air temperature were monitored with a digital anemometer. The uniformity of application varied between 71.6 and $82.0 \%$, being considered reasonable. The average Eap was $87.1 \%$. The pressure of $6.0 \mathrm{kgf} \mathrm{cm}-2$ provided better performance in uniformity and efficiency. 


\section{INTRODUÇÃO}

A cultura de cana-de-açúcar Saccharum officinarum L., é uma planta pertencente à família Poaceae, que surgiu na Oceania e na Ásia. É uma das culturas agrícolas de maior importância para Brasil é cultivada principalmente nas regiões Centro Sul, onde apresentam bom desenvolvimento e alta produtividade (SANTOS et al., 2011; ALENCAR, 2012). É uma das primeiras culturas introduzida no Brasil e se adaptou com muita facilidade as condições climáticas do local. Na safra 2017/2018 o Brasil foi o maior produtor mundial com a produção de 633,26 milhões de toneladas de cana-de-açúcar, onde foi cultivado aproximadamente 8,8 milhões de hectares da cultura, segundo CONAB (2018).

A produção de cana-de-açúcar está diretamente associada a condições climáticas e disponibilidade hídrica. O clima tropical, quente e úmido, temperatura entre $19^{\circ}$ e $32^{\circ} \mathrm{C}$ e com chuvas bem distribuídas, precipitação acumulada acima de 1000 milímetros ao ano, são fatores climáticos favoráveis para o seu desenvolvimento. A cultura tem duas principais fases de desenvolvimento, a de crescimento vegetativo, que é favorecida pelo clima quente e úmido, e a fase de maturação da cultura, quando o acúmulo de sacarose é favorecido por temperaturas mais amenas e com baixa disponibilidade hídrica (AGEITEC, 2019; CAETANO, 2017).

Segundo ANA (2017), a agricultura é uma das atividades que mais utiliza água, representado por $46,2 \%$ da retirada em mananciais $\left(969 \mathrm{~m}^{3} \mathrm{~s}^{-1}\right)$, já o abastecimento urbano e responsável por 23,3\% do consumo, e em terceiro a geração de energia termelétrica fica com 10,3\%.
A uniformidade da lâmina de aplicação é o principal critério utilizado para avaliar qualquer sistema de irrigação. Segundo Bernardo et al. (2009), para a cultura da cana-de-açúcar, a irrigação pode ser feita em dois modos, irrigação complementar que é de salvamento onde o uso da água é mínimo no estágio inicial da cultura e a irrigação suplementar voltado para o aumento da produtividade. O número de cortes nos canaviais e o aumento da produtividade estão relacionados diretamente com a implantação de sistemas de irrigação na cultura.

De acordo com Frizzone et al. (2001), a irrigação em cana-de-açúcar em são de grande importância econômica, onde tem grande influência em alguns fatores na cultura, podendo aumentar a longevidade do canavial e obter maior produtividade. Quando a tecnologia de irrigação não é bem utilizada e se tem um ganho menor que 0,100 t/mm, a irrigação chega a ser inviabilizada.

As áreas irrigadas com carreadores entre os carretéis com maior espaçamento até $68 \mathrm{~m}$, apresentam um aumento de 13,33\% em área irrigada por faixa, onde a velocidade do vento não ultrapasse $2,2 \mathrm{~m} / \mathrm{s}$, recomendado para equipamento de irrigação autopropelido (ROCHA et al., 2005). Um fator muito importante a ser observado é a sobreposição das lâminas, que não pode ser menor que $80 \%$ do diâmetro molhado pelo aspersor.

A uniformidade da lâmina de irrigação pode ser mais precisa dependendo do ângulo de giro do canhão no momento da irrigação. Onde um ângulo de $210^{\circ}$ a $240^{\circ}$ se tem uma melhor uniformidade com o uso de aspersores PLONA-RL250 bocal de $6 \mathrm{~mm}$ (PADRO et al., 2007). Com o aumento da 
velocidade do vento, o CUE diminui pressões de trabalho, considerando a ação do vento proporcionalmente ao aumento do espaçamento e da temperatura na distribuição da lâmina de entre aspersores. Com pressões de trabalho mais irrigação.

altas, aumenta a uniformidade no espaço de pressão estudado que é de 318 a $729 \mathrm{KPa}$ (AZEVEDO et al., 2000). Já Conceição (2017), fez um estudo onde o vento interferiu diretamente na distribuição da água no momento da irrigação. $\mathrm{O}$ tamanho da gota é uma forma de minimizar esses aspectos, principalmente por derivas, onde o aumento da velocidade do vento favorece essa ocorrência.

Diante disso objetivou-se avaliar a uniformidade da lâmina aplicada com diferentes

\section{MATERIAL E METODOS ÁREA EXPERIMENTAL}

O presente trabalho foi desenvolvido no dia 14 de setembro de 2018, na Fazenda Barra Alegre, no município de Santa Isabel - GO, localizada nas coordenadas de Latitude: $15^{\circ}$ 7'36.22"S e Longitude: 49²0'57.28"O (Figura 01). Nesta área é cultivada a cultura da cana-de-açúcar onde se utiliza irrigação por sistema de aspersão autopropelido.

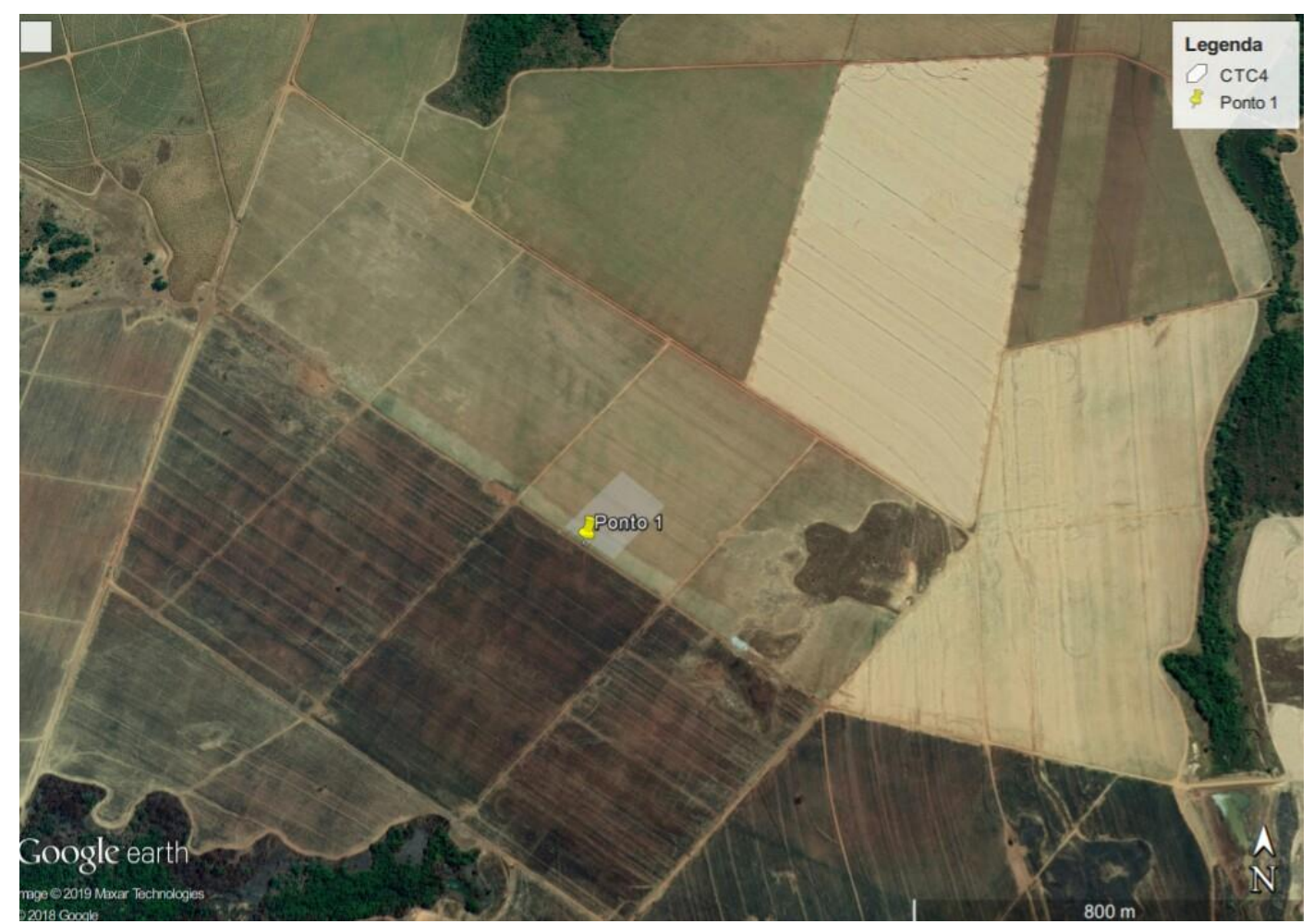

Figura 01: Mapa de localização da avaliação de uniformidade em irrigação por autopropelido. Fonte: Google Earth.

\section{SISTEMA DE IRRIGAÇÃO}

Os carretéis foram dispostos em paralelo com espaçamento de $66 \mathrm{~m}$. Cada carretel foi regulado para recolher em uma velocidade média de $100 \mathrm{~m} / \mathrm{h}$, com a pretensão de aplicar uma lâmina de $16,33 \mathrm{~mm}$ no total, sendo $8,16 \mathrm{~mm}$ cada equipamento (Figura 02). 


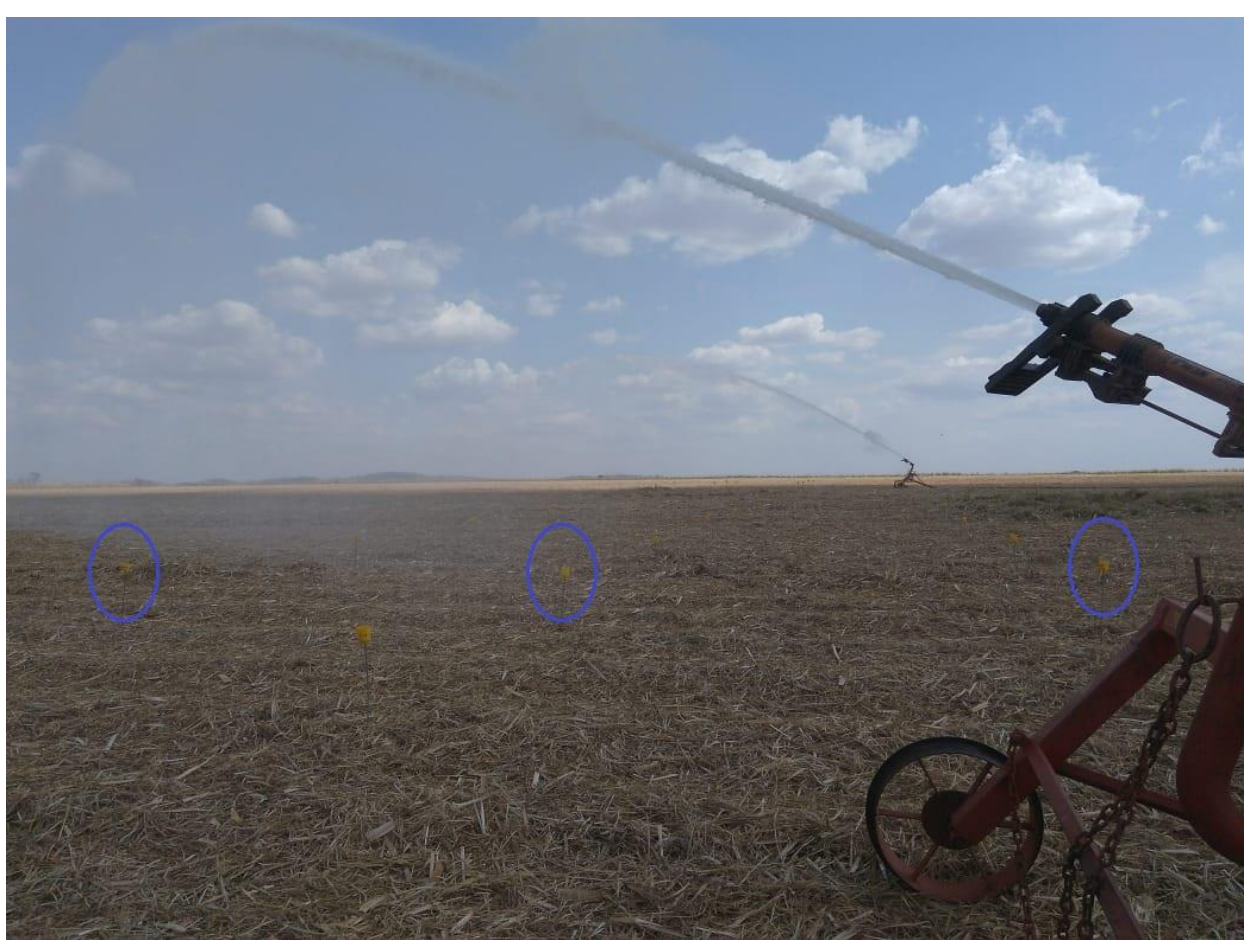

Figura 02: Sistema de irrigação por aspersão autopropelido em operação para o ensaio de uniformidade. Em detalhe os coletores.

Os carretéis de irrigação são da marca irrigação com bocal de $35 \mathrm{~mm}$ e a vazão de 108,1 IRRIGABRASIL, modelo TURBOMAQ $\mathrm{m}^{3} \mathrm{~h}^{-1}$. No Quadro 1 estão descritos os dados 140/GS-6R, comprimento da mangueira é de 300 técnicos do equipamento. metros com diâmetro de $140 \mathrm{~mm}$, o canhão de

Quadro 1: Dados técnicos do carretel irrigador.

\begin{tabular}{|c|c|}
\hline \multicolumn{2}{|l|}{ DADOS TÉCNICOS } \\
\hline Marca & Irrigabrasil \\
\hline Modelo & Turbomaq140 \\
\hline Tubo de polietileno & $140 \mathrm{~mm}$ \\
\hline Comprimento tubo de polietileno & $300 \mathrm{~m}$ \\
\hline Pressão de entrada na maquina & 8,3 a $9,0 \mathrm{kgf} / \mathrm{cm} 2$ \\
\hline Vazão & $108,1 \mathrm{~m} 3 / \mathrm{h}$ \\
\hline Aspersor & Setorial Twin 202 plus \\
\hline Bocal & $35 \mathrm{~mm}$ \\
\hline
\end{tabular}

\section{ENSAIO DE UNIFORMIDADE}

Foram instalados copos coletores de precipitação no espaçamento entre dois carretéis. Os copos coletores são da marca Fabrimar, com área de coleta igual a $50,24 \mathrm{~cm}^{2}$, foram instalados aproximadamente $0,60 \mathrm{~m}$ do solo, suspensos por hastes de alumínio, com um espaçamento de $5 \mathrm{~m}$ entre coletor e $5 \mathrm{~m}$ entre linhas, totalizando 13 coletores por linha e três linhas, foram realizado apenas uma repetição de cada ensaio, sendo o primeiro com $06 \mathrm{kgf} \mathrm{cm}^{-2}$ na entrada do equipamento e $04 \mathrm{kgf} \mathrm{cm}^{-2}$ na saída, o segundo com 
$07 \mathrm{kgf} \mathrm{cm}^{-2}$ na entrada do equipamento e $05 \mathrm{kgf}$ polietileno do autopropelido, em um velocidade de

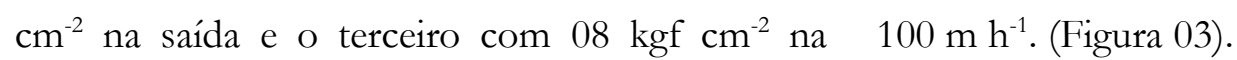

entrada e $06 \mathrm{kgf} \mathrm{cm}^{-2}$ na saída, cada ensaio teve a duração de 40 minutos, onde era percorrido 67 metros linear de recolhimento do do tubo de

No decorrer de cada tratamento foram realizado a medição da temperatura do $\operatorname{ar}\left({ }^{\circ} \mathrm{C}\right)$ e da velocidade do vento (m s-1) foram medidos a cada dez minutos ao longo de cada teste, utilizando Termo - Anemômetro digital HDA - 910 da marca HIKARI.

Figura 03. Esquema da disposição dos coletores e dos carretéis para realização do teste de uniformidade.

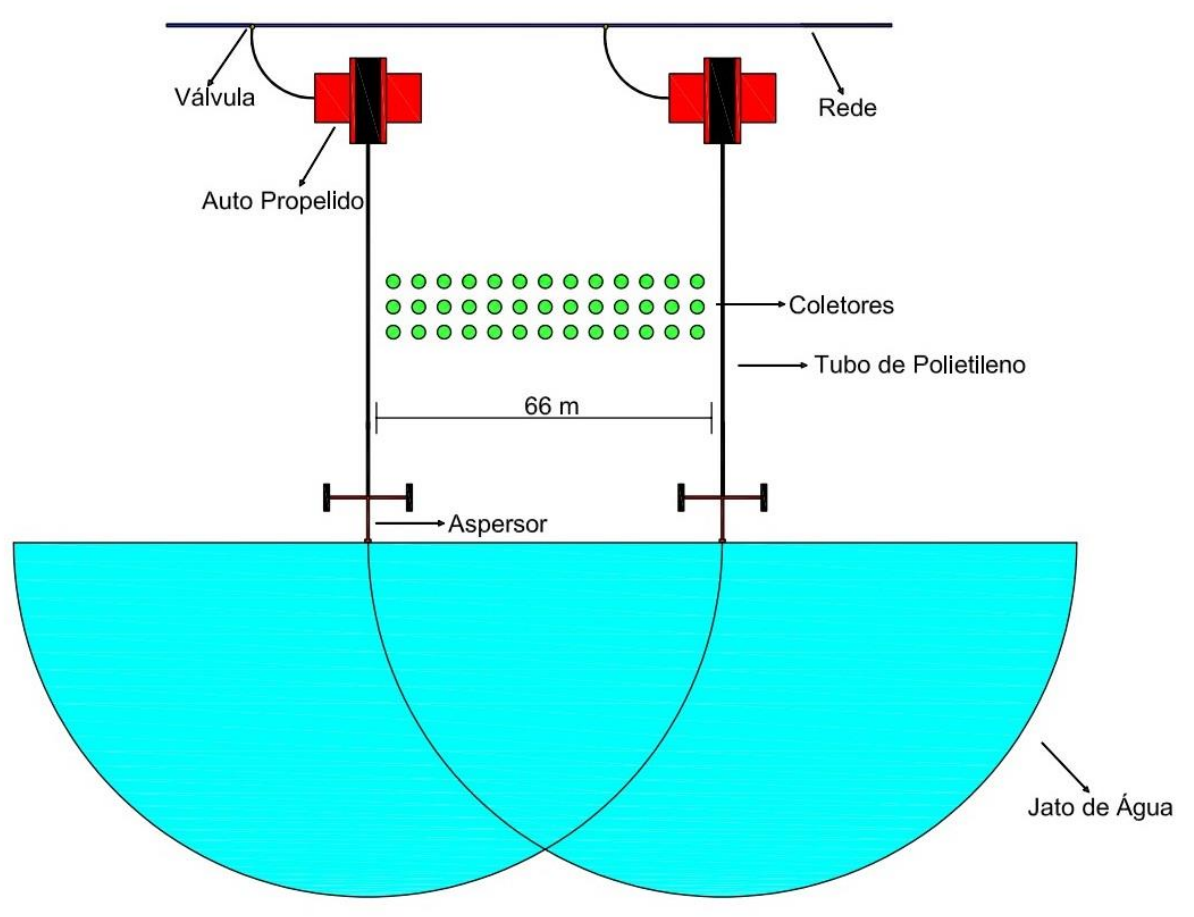

A uniformidade de aplicação foi de água observada em dois coletores instalados na monitorada com três diferentes pressões de serviço adjacência da área irrigada, cada coletor continha no bocal: 4,0 kgf cm $\mathrm{kg}^{-2}, 5,0 \mathrm{kgf} \mathrm{cm}{ }^{-2}$ e $6,0 \mathrm{kgf} \mathrm{cm}^{-2}$. inicialmente $100 \mathrm{~mL}$ de água.

Seguindo a regulagem de recolhimento igual para Os ensaios ocorreram no dia 14 de todos. No decorrer de cada ensaio foi quantificada setembro de 2018 (Tabela 2). Os testes foram a evaporação da água pela ação da temperatura e realizados no período da tarde.

do vento por meio da variação média do volume 
Tabela 2. Descrição dos ensaios de uniformidade de aplicação de água em sistema de aspersão autopropelido.

\begin{tabular}{|c|c|c|c|c|c|c|c|}
\hline \multirow{2}{*}{ 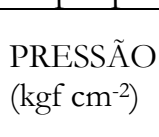 } & \multirow{2}{*}{$\begin{array}{l}\text { HORÁRIO } \\
\text { INÍCIO }\end{array}$} & \multirow{2}{*}{$\begin{array}{l}\text { TEMPO DE } \\
\text { DURAÇÃO } \\
\text { (min.) }\end{array}$} & \multirow{2}{*}{$\mathrm{T}\left({ }^{\circ} \mathrm{C}\right)$} & \multirow{2}{*}{$\mathrm{E}(\mathrm{mm})$} & \multicolumn{3}{|c|}{$\begin{array}{l}\text { VELOCIDADE DO VENTO } \\
\left(\mathrm{m} \mathrm{s}^{-1}\right)\end{array}$} \\
\hline & & & & & MÁXIMA & MÍNIMA & MÉDIA \\
\hline 4,0 & $12: 25$ & 40 & 37,28 & 2,5 & 4,0 & 1,5 & 3,0 \\
\hline 5,0 & $13: 30$ & 40 & 35,76 & 1,5 & 7,8 & 2,7 & 4,9 \\
\hline 6,0 & $14: 20$ & 40 & 35,74 & 1,4 & 4,9 & 2,0 & 3,5 \\
\hline
\end{tabular}

A partir do volume retido nos coletores, foram calculados os coeficientes de uniformidade e eficiência descritos a seguir.

- Coeficiente de Uniformidade Estatístico de Wilcox \& Swailes (CUE).

$$
C U E=100 \cdot\left(1-\frac{S d}{\bar{X}}\right)
$$

CUE $=$ Coeficiente de Uniformidade Estatístico, em \%;

$\mathrm{Sd}=$ Desvio-padrão dos valores de precipitação, em mm;

$\mathrm{X}=$ Lâmina média de todas as observações, em mm.

- Coeficiente de Uniformidade de Christiansen (CUC)

$C U C=100 \cdot\left(1-\frac{\sum_{i=1}^{n}|X i-\bar{X}|}{n \cdot \bar{X}}\right)$

CUC = Coeficiente de Uniformidade de Christiansen, em \%;

$\mathrm{Xi}=$ Lâmina medida no coletor, $\mathrm{em} \mathrm{mm}$;

$\mathrm{n}=$ número de coletores.

- Coeficiente de Uniformidade de Distribuição (CUD)

$$
C U D=100 \cdot\left(\frac{X_{25 \%}}{\bar{X}}\right)
$$

$\mathrm{X}_{25 \%}=$ média de $25 \%$ das observações com menores valores.
- A eficiência de aplicação foi determinada por:

$$
\begin{aligned}
E A p & =100 \cdot \frac{X_{\text {aplicada }}}{X_{\text {pretendida }}} \\
\text { EAp } & =\text { eficiência de aplicação em \%. }
\end{aligned}
$$

\section{RESULTADOS E DISCUSSÃO CONDIÇÕES METEOROLÓGICAS}

A média da temperatura do ar no decorrer do primeiro ensaio foi de $37,28{ }^{\circ} \mathrm{C}$ e a velocidade média do vento de $3,02 \mathrm{~m} \mathrm{~s}^{-1}$. No segundo ensaio, a média da temperatura avaliada foi de $35,75^{\circ} \mathrm{C}$ e a velocidade média do vento foi de $4,92 \mathrm{~m} \mathrm{~s}^{-1}$, já no terceiro ensaio, a média da temperatura do ar avaliada foi de $35,74{ }^{\circ} \mathrm{C}$ e a velocidade média do vento foi de $3,47 \mathrm{~m} \mathrm{~s}^{-1}$.

Nos Gráficos 1, 2 e 3 constata-se que a temperatura teve uma oscilação de acordo com a velocidade do vento, podendo observar que durante o tempo de duração de cada experimento, que quando a velocidade do vento se elevada a temperatura reduzia. As temperaturas permaneceram acima de $33^{\circ} \mathrm{C}$ nos três ensaios.

Velocidade do vento ideal para irrigar estando abaixo de $2 \mathrm{~m} \mathrm{~s}^{-1}$, valor máximo preconizado pela norma de ensaio (ABNT, 1999). 
Gráfico 1: Temperatura e velocidade do vento. Pressão $\left(4,0 \mathrm{kgf} \mathrm{cm}^{-2}\right)$.

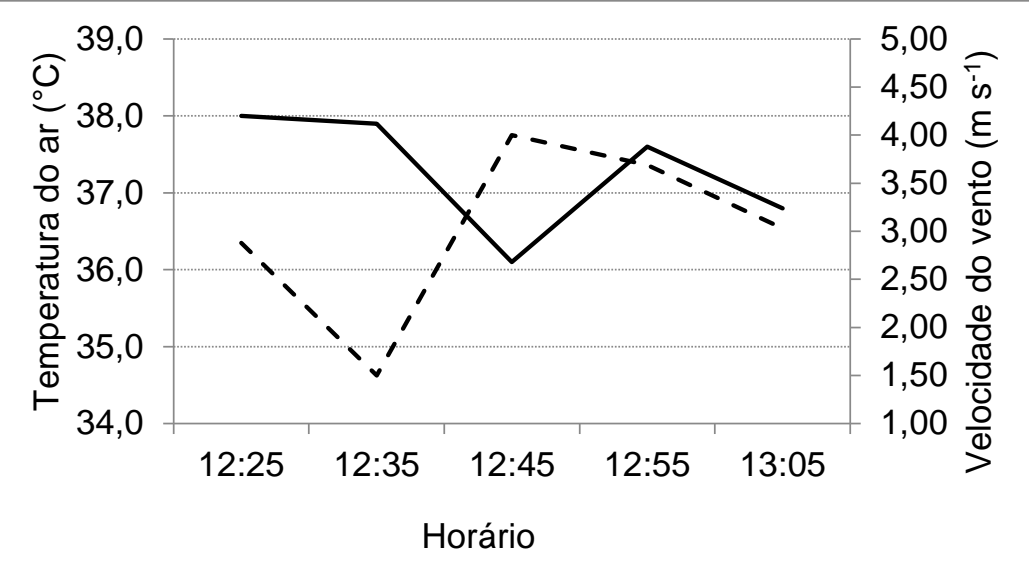

— Temperatura do ar _ _ - Velocidade do vento

Gráfico 2: Temperatura e velocidade do vento. Pressão $\left(5,0 \mathrm{kgf} \mathrm{cm}^{-2}\right)$.

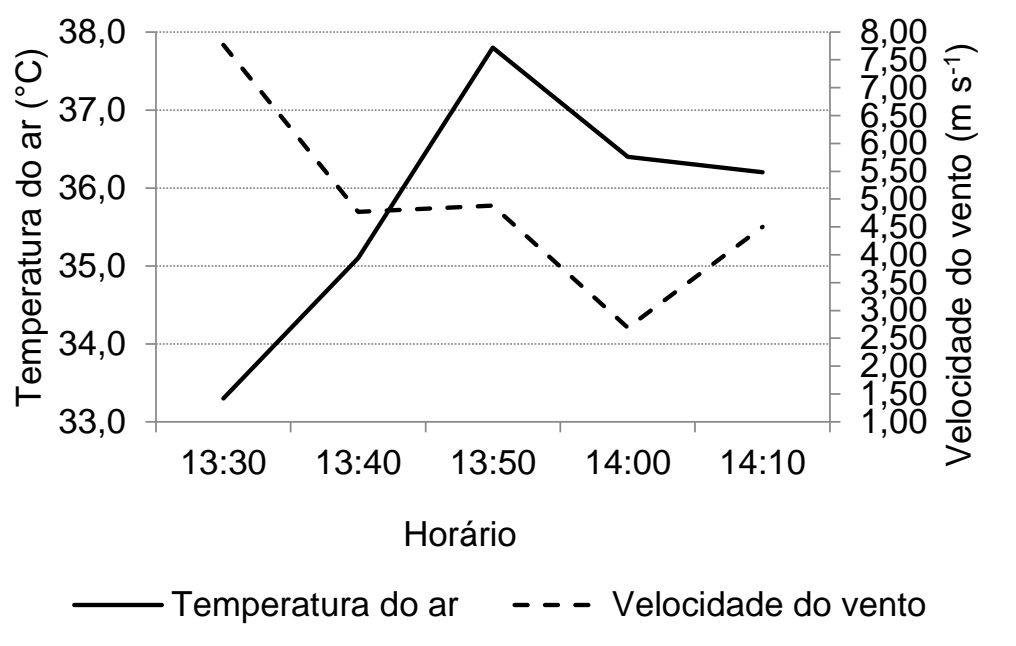

Gráfico 3: Temperatura e velocidade do vento. Pressão $\left(6,0 \mathrm{kgf} \mathrm{cm}^{-2}\right)$.

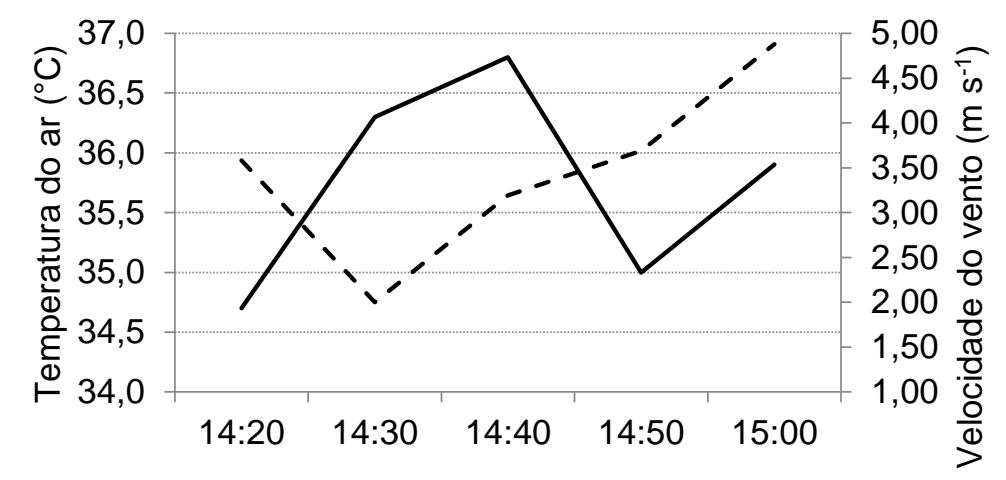

Horário

— Temperatura do ar _ _ - Velocidade do vento

A velocidade do vento durante os ensaios foi superior no horário entre 13:30 e 13:40 horas, coincidindo com o ensaio com pressão de 5,0 kgf $\mathrm{cm}^{-2}$. Consequentemente no gráfico 4 , mostra-se direção. que a uma maior variação das lâminas em uma das extremidades, comparada com a outra. Este fatos pode estar relacionado a velocidade do vento e sua 


\section{COEFICIENTES DE UNIFORMIDADE}

$\mathrm{Na}$ Tabela 3 estão apresentados os dados dos coeficientes de uniformidade dos carretéis em estudo (CUE, CUC, CUD e Eap). Pode-se observar que os ensaios com a pressão $6,0 \mathrm{kgf} \mathrm{cm}^{-}$ ${ }^{2}$ alcançaram os coeficientes de uniformidade mais elevados, onde para o CUE é classificado como “razoável” baseando-se na metodologia adaptada por Mantovani (2001). Seguindo o mesmo parâmetro de avaliação, o CUC nos ensaios com
$4,0 \mathrm{kgf} \mathrm{cm} 2$ e $6,0 \mathrm{kgf} \mathrm{cm}^{-2}$, são classificados como "bom". Onde o ensaio com 5,0 $\mathrm{kgf} \mathrm{cm}^{-2}$ como "razoável", pode ser justificado pela maior velocidade do vento que foi superior em relação aos demais ensaios, Tabela 2. Já para o CUD todos são classificados como "bom”. A Eap em todos os ensaios ficaram em um padrão aceitável, acima de 86\%. De acordo com Pair et al. (1969), a eficiência total de aplicação de água em sistemas por aspersão varia de 70\% em regiões quentes e áridas, a 85\% em regiões úmidas e frias.

Tabela 3. Coeficientes de uniformidade e eficiência avaliados para sistema de irrigação autopropelido com diferentes pressões de serviço.

\begin{tabular}{llll}
\multirow{2}{*}{ Parâmetro avaliado } & \multicolumn{3}{l}{ Pressão de Serviço $\left(\mathrm{kgf} \mathrm{cm}^{-2}\right)$} \\
\cline { 2 - 4 } & 4,0 & 5,0 & 6,0 \\
\hline CUE $(\%)$ & 71,59 & 73,20 & 77,48 \\
CUC (\%) & 80,61 & 78,85 & 82,03 \\
CUD (\%) & 75,68 & 72,03 & 77,60 \\
Eap (\%) & 87,51 & 86,16 & 87,57 \\
\hline
\end{tabular}

De acordo com o gráfico com gráfico 4, obtida e a lâmina de pretendida. Observamos que onde estão apresentados o perfil de distribuição de o ensaio com a pressão $06 \mathrm{kgf} \mathrm{cm}-2$ obteve melhor água ao longo da faixa irrigada, a lâmina média uniformidade em ralação a lamina esperada.

Gráfico 4: Lâmina aplicada ao longo da faixa irrigada, em função da pressão de serviço do equipamento de irrigação.

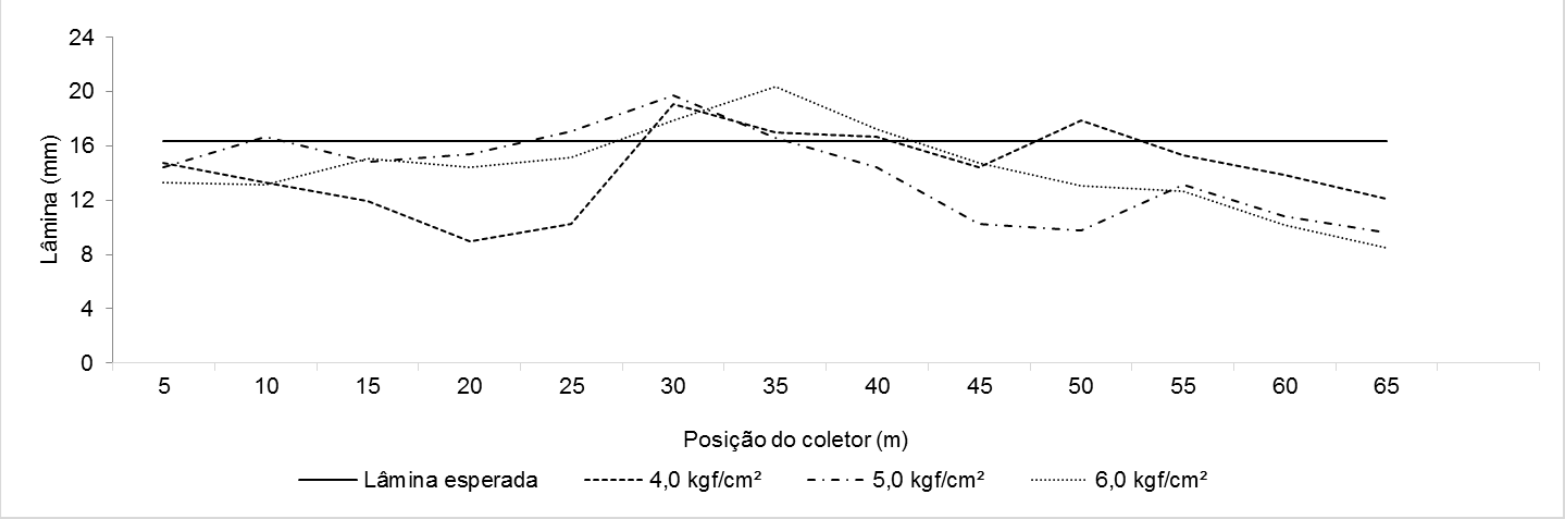

Para o ensaio com 4,0 kgf $\mathrm{cm}^{-2}$, a lâmina observar-se que a velocidade média do vento $(3,0$ média aplicada foi de $14,29 \mathrm{~mm}$. Podendo $\mathrm{m} \mathrm{s}^{-1}$ ) foi inferior que os demais ensaio, 
conseguintemente a lâmina aplicada $(14,29 \mathrm{~mm})$ ficou entre os valores encontrados nos demais ensaios.

Para o ensaio com 5,0 kgf $\mathrm{cm}^{-2}$, a lâmina média aplicada foi de $14,07 \mathrm{~mm}$, onde constatou que nos coletores de uma das extremidades, observou-se oscilação mais acentuada em relação a lâmina média quando comparado com os observados na outra extremidade. Podendo-se justificar essa oscilação pela condição climática no momento do desenvolvimento do ensaio, onde a velocidade do vento foi superior, Tabela 2. Consequentemente a lâmina aplicada foi a menor comparada com os demais ensaios.

Para o ensaio com $6,0 \mathrm{kgf} \mathrm{cm}^{-2}$ a lâmina aplicada foi de $14,30 \mathrm{~mm}$. Ao observar os perfis de uniformidade de aplicação das lâminas, nota-se que a menor variação de distribuição da lâmina irrigada foi no ensaio com a pressão de $6,0 \mathrm{kgf} \mathrm{cm}^{-2}$, quando comparado com os demais. Isso pode ser justificado devido a pressão ter garantido que o alcance do jato de água fizesse melhor sobreposição.

Em relação a maior lâmina aplicada que foi com a pressão $6,0 \mathrm{kgf} \mathrm{cm}^{-2}$, houve uma redução de $0,07 \%$ para a pressão $4,0 \mathrm{kgf} \mathrm{cm}^{-2}$ e de 1,6\% para pressão $5,0 \mathrm{kgf} \mathrm{cm}{ }^{-2}$.

De acordo com Lopes et al. (2014) ao realizar um trabalho onde utilizaram irrigação por aspersão convencional, e obtiveram CUE (\%) considerado foi satisfatório de $94,48 \%$, segundo os autores, este valor foi superior ao encontrado na literatura. Observa-se que Lopes et al. (2014), obtiveram valor satisfatório com o uso desse método de irrigação comparado com o uso de autopropelido, porém pode ser justificado este valor, devido o experimento do mesmo ter sido conduzido em uma área experimental, em canteiros e sem a interferência do vento, e com um sistema diferente. Já os valores de CUD e CUC se encontram abaixo do ideal, 46,38\% e 55,97\%. Segundo o autor a falta de informações técnicas de como foi feito o dimensionamentos dos aspersores e pressões ideal de trabalho, pode ter comprometido a eficiência da uniformidade.

Em um sistema de irrigação por aspersão em pastagem, Pereira et al. (2016), encontrou os seguintes valores para CUD 69,79\% sendo classificado como bom, CUC 79,31\% classificado com razoável e Us coeficiente estatístico 75,41\% classificado como razoável, por (Mantovani 2001). Em contra partida (Paulino et al. 2009), avaliou um sistema de irrigação por aspersão convencional em várias propriedades, encontrou na propriedade “A" CUC 80,20\% considerado como bom, CUD 68,97\% como ruim e CUE 76,60\% considerado como regular. Na propriedade "B" CUC 74,10\%, CUE $72,00 \%$ foram classificados como regulares e CUD 52,19\% inaceitável. $\mathrm{Na}$ propriedade "C" CUC 78,62\%, CUE 77,00\% classificado como regular e CUD 69,43\% considerado ruim. Já na propriedade "D" CUC 51,41\%, CUD 27,78\% CUE $44,00 \%$ todos os coeficientes foram classificados como inaceitável, abaixo de 60\%, segundo a classificação de Bernardo et al. (2009). Para (Ea) eficiência de aplicação, todos os sistema foram classificados como inaceitáveis.

A correlação linear de Pearson, na Tabela 4, permite observar claramente o efeito negativo da velocidade do vento (Vmax, Vmed e Vmin) sobre a uniformidade de aplicação. Além disso, observase que a temperatura é um fator que colaborou 
fortemente para as perdas por evaporação, justificando a maior evaporação no ensaio que ocorreu com maior temperatura do ar.

Tabela 4. Coeficiente de correlação linear de Pearson para as variáveis meteorológicas e os coeficientes de uniformidade e eficiência.

\begin{tabular}{|c|c|c|c|c|c|c|c|c|c|c|c|}
\hline & $E(\mathrm{~mm})$ & Tmax & Tmed & Tmin & $V \max$ & $V$ med & $V \min$ & CUE & CUC & CUD & $E A$ \\
\hline $\begin{array}{l}\mathrm{E} \\
(\mathrm{mm})\end{array}$ & 1 & & & & & & & & & & \\
\hline Tmax & 0,69 & 1 & & & & & & & & & \\
\hline Tmed & 1,00 & 0,64 & 1 & & & & & & & & \\
\hline Tmin & 0,82 & 0,16 & 0,86 & 1 & & & & & & & \\
\hline $\mathrm{V} \max$ & $-0,62$ & 0,14 & $-0,67$ & $-0,96$ & 1 & & & & & & \\
\hline $\mathrm{V}$ med & $-0,62$ & 0,14 & $-0,67$ & $-0,96$ & 1,00 & 1 & & & & & \\
\hline Vmin & $-0,77$ & $-0,06$ & $-0,81$ & $-1,00$ & 0,98 & 0,98 & 1 & & & & \\
\hline CUE & $-0,77$ & $-0,99$ & $-0,72$ & $-0,26$ & $-0,03$ & $-0,03$ & 0,17 & 1 & & & \\
\hline CUC & $-0,02$ & $-0,74$ & 0,05 & 0,55 & $-0,77$ & $-0,77$ & $-0,63$ & 0,66 & 1 & & \\
\hline CUD & 0,09 & $-0,65$ & 0,17 & 0,65 & $-0,84$ & $-0,84$ & $-0,71$ & 0,57 & 0,99 & 1 & \\
\hline EA & 0,39 & $-0,39$ & 0,46 & 0,85 & $-0,97$ & $-0,97$ & $-0,89$ & 0,29 & 0,91 & 0,95 & 1 \\
\hline
\end{tabular}

Segundo LOPES et al. (2014), ao avaliar um sistema de irrigação por aspersão convencional encontrou os seguintes resultado, valores de CUD e CUC ficaram abaixo do ideal, onde o valor de CUC é inaceitável pelo sistema avaliado. Já o CUE foi satisfatórios, classificado na faixa de excelente.

Segundo FEREITAS et al. (2009), a irrigação deve ser utilizada para que a cultura da cana-de-açúcar expresse todo seu potencial produtivo, e não como um seguro contra as secas e os veranicos, podendo ter uma redução de insumos através da fertirrigação. Para que o projeto de irrigação tenha um boa uniformidade de irrigação e seja eficiente, é importante o uso de materiais de boa qualidade e com o cálculos hidráulicos precisos.

Déficit hídrico afeta negativamente o crescimento e desenvolvimento da parte vegetativa do canavial, acelerando a senescência foliar e da planta como um todo, levando a uma redução na interceptação da radiação solar, na eficiência do uso de água e a eficiência da fotossíntese é prejudicada, Campos et al. (2013).

\section{CONCLUSÃO}

A uniformidade de aplicação das lâminas de irrigação foram classificadas entre razoável e bom.

Dentre as pressões avaliadas a pressão de 6,0 $\mathrm{kgf} \mathrm{cm}^{-2}$ foi a que apresentou melhor uniformidade e eficiência.

A eficiência de aplicação média do sistema foi de $87,1 \%$.

A variação da lâmina média aplicada, considerando as diferentes pressões, foi de no máximo $1,6 \%$.

Os resultados obtidos servem como base para o manejo da irrigação utilizando o aspersor autopropelido, recomendando-se a reavaliação periódica da uniformidade em lâminas aplicadas a 
fim de monitorar a distribuição de água para a cultura irrigada, bem como identificar pontos de déficit hídrico em potencial.

\section{REFERÊNCIAS BIBLIOGRÁFICAS}

ABNT - Associação Brasileira de Normas Técnicas. Equipamentos de irrigação agrícola: Aspersores rotativos. Parte 1: Requisitos para projetos e operação. Projeto 04:015.08-012. Parte 2: Uniformidade de distribuição e métodos de ensaio. Projeto 04:015.08-013. Rio de Janeiro: ABNT, 1999. 22p.

AGEITEC. Agência Embrapa de Informação Tecnológica.

$<$ Http://www.agencia.cnptia.embrapa.br/g estor/cana-de-açucar/arvore/

CONTAG01_ 20_3112006152934.html>, Acesso em: 27 de maio de 2019.

ALENCAR K. Análise do balanço entre demanda por etanol e oferta de cana de açúcar no brasil. Fundação Getúlio Vargas, Escola de Economia de São Saulo, Universidade de São Saulo, Escola de Agricultura Luiz de Queiroz, Empresa Brasileira de Pesquisa Agropecuária. São Paulo 2012, p. 12-49.

ANA - AGÊNCIA NACIONAL DAS AGUAS. Conjuntura dos recursos hídricos no Brasil 2017. Relatório pleno. Brasília: ANA, 169 p. 2017.

AZEVEDO H. J.; BERNARDO S.; RAMOS M. M.; SIDIYAMA G. C.; CECON P. R. Influência de fatores climáticos $\mathrm{e}$ operacionais sobre a uniformidade de distribuição de água, em um sistema de irrigação por aspersão de alta pressão. $R$. Bras. Eng. Agríc. Ambiental, Campina Grande, v.4, n.2, p.152-158, 2000.

BERNARDO, S.; SOARES, A. A.; MANTOVANI, E. C. Manual de irrigação. 8. ed. Viçosa, MG: UFV, 2009. 625p.
CAETANO J. M. Modelagem agrometeorológica da cana-de-açúcar nas condições edafoclimáticas do centro-oeste goiano. Universidade Federal de Goiás, Escola de Agronomia, Programa de Pós-graduação em Agronomia. P. 16 125. Dezembro de 2017.

CAMARGO, D. C. Curso de avaliação de equipamento de irrigação. Fortaleza: INOVAGRI/IFCE, 28 p., 2016.

CONAB. Companhia Nacional de Abastecimento

$<$ https://www.conab.gov.br/infoagro/safras/cana/>, Acesso em: 27 de maio de 2019.

CONCEIÇÃO M. A. F. Simulação da distribuição de água em microaspersores sob condição de vento. Piracicaba, Estado de São Paulo - Brasil Julho - 2002.

FRIZZONE J. A.; MATIOLI C. S.; REZENDE R.; GONÇALVES A. C. A. Viabilidade econômica da irrigação suplementar da cana-de-açúcar, Saccharum spp., para a região Norte do Estado de São Paulo. Maringá, v. 23, n. 5, p. 1131-1137, 2001.

LOPES M. A. P.; MORAIS E. G.; RESENDE C. P.; VAZ G. H. B.; FARIA R. B.; TORMEN G. P.; Avaliação de uniformidade de aplicação de água de um sistema de irrigação por aspersão convencional no setor de olericultura do IFMG, campusBambuí. Bambuí, MG. IFMG, 2014.

MANTOVANI, E.C. Avalia: Programa de Avaliação da irrigação por aspersão e localizada. Viçosa, MG. UFV, 2001.

PAIR, C. H.; WALTER, W. H.; CRAWFOR, D. R; KENNITH, R. F. Sprinkler

irrigation. Washington, D. C. Sprinkler Association, 1969.444p.

PAULINO M. A. O.; FIGUEREDO F. P.; FERNANDES R. C.; MARIA J. T. L. S.; GUILHERME D. O.; BARBOSA F. S. Avaliação da uniformidade e eficiência de aplicação de água em sistemas de 
irrigação por aspersão convencional. Revista Brasileira de Agricultura Irrigada v.3, n.2, p.48-54, 2009.

PADRO G.; COLOMBO A. Análise da uniformidade de aplicação de água pelo aspersor plona-rl250 em sistemas autopropelidos de irrigação. Irriga, Botucatu, v. 12 , n. 2, p. 259-262, abril-junho, 2007.

PEREIRA L. R.; SOUZA J. M.; RAFAEL. M. CRUZ E. A.; TEIXEIRA A. G.; REIS E. F. Uniformidade e eficiência de aplicação de água em um sistema de irrigação por aspersão em pastagem. Revista Agrarian, v.9, n.32, p. 156-161, Dourados, 2016.
PEREIRA R. M.; JOSÉ A. J.; CASAROLI D.; SALES D. L.; RODRIGUEZ W. D. M.; SOUZA J. M. F. Viabilidade econômica da irrigação de cana-de-açúcar no cerrado brasileiro. Irriga, Botucatu, Edição Especial, IRRIGA \& INOVAGRI, p. 149157, 2015.

ROCHA, F. A.; PEREIRA, G. M.; ROCHA, F. S.; SILVA, J. O. Análise da uniformidade de distribuição de água de um equipamento autopropelido. Irriga, Botucatu, v. 10, n. 1, p. 96-106, janeiro-abril, 2005.

SANTOS F.; BORÉM A.; CALDAS C. Cana-deaçúcar: bioenergia, açúcar e etanol: tecnologia e perspectivas. - 2. Ed. E ampl. - Viçosa, MG: Os editores, 2011. p. 25-28. 